\title{
Lamb Waves Decomposition and Mode Identification Using Matching Pursuit Method
}

\author{
Buli Xu, Victor Giurgiutiu, Lingyu Yu \\ Mechanical Engineering Department, University of South Carolina \\ Columbia, SC 29208 \\ xub@engr.sc.edu, giurgiut@engr.sc.edu, yu3@engr.sc.edu
}

\begin{abstract}
Matching pursuit (MP) is an adaptive signal decomposition technique and can be applied to process Lamb waves, such as denoising, wave parameter estimation, and feature extraction, for health monitoring applications. This paper explored matching pursuit decomposition using Gaussian and chirplet dictionaries to decompose/approximate Lamb waves and extract wave parameters. While Gaussian dictionary based MP is optimal for decomposing symmetric signals, chirplet dictionary based MP is able to decompose asymmetric signals, e.g., dispersed Lamb wave. The extracted parameter, chirp rate, from the chirplet MP can be used to correlate with two Lamb wave modes, $\mathrm{S}_{0}$ and $\mathrm{A}_{0}$.
\end{abstract}

Keywords: Lamb wave, matching pursuit decomposition, Gabor dictionary, chirplet dictionary, structural health monitoring, Piezoelectric wafer active sensors, PWAS

\section{INTRODUCTION}

In recent years, a large number of papers have been published on the use of Lamb waves for nondestructive evaluation and damage detection for structural health monitoring (SHM) applications. The benefits of guided waves over other ultrasonic methods is due to their: (1) variable mode structures and distributions; (2) multimode charactesr; (3) propagation for long distances; (4) capability to follow curvature and reach hidden and /or buried parts; (5) sensitivity to different type of flaws. Some modes (e.g. $\mathrm{A}_{0}$ mode) are sensitive to surface defects and some modes (e.g., $\mathrm{S} 0$ mode) are sensitive to internal defects. Displacement fields across the experiment wave structure thickness can explain the sensitivity of lamb modes to defect types (Pan et. al., 1999; Edalati et. al., 2005; Giurgiutiu 2008). Properly identification of Lamb wave modes and tracking the change of a certain mode is of great significance for SHM applications.

The objective of this paper is to explore the application of matching pursuit to decompose and approximate Lamb waves using two types of dictionaries, i.e., Gabor dictionary and chirplet dictionary, and to demonstrate the capabilities of this method to identify low-frequency Lamb wave modes ( $\mathrm{S}_{0}$ and $\mathrm{A}_{0}$ modes) and other wave parameters, such as central frequency, time-of-flight, etc., that are useful for structural health monitoring applications.

\section{SIGNAL DECOMPOSITION}

The purpose of signal decomposition is to extract a set of features characterizing the signal of interest. This is usually realized by decomposing the signal on a set of elementary functions (Lankhorst 1996) (Durka 2007). A widely used signal decomposition method is Fourier transform, which decomposes signals on a series of harmonic functions. However, the harmonics basis functions have global support. For example, to decompose a signal with a presence of a discontinuity in time, all the weights of the basis functions will be affected; the phenomenon of discontinuity is diluted. Therefore, Fourier transform is usually used for stationary signals. To better characterize a signal with time-varying nature, basis functions that are localized both in time and frequency are desired. This gives rise to time-frequency decomposition methods, including short time Fourier transform (STFT), wavelet transform, Wigner-Ville distribution, matching pursuit decomposition, etc. 


\subsection{Time-Frequency Decomposition}

For STFT, the signal is multiplied with a window function to delimit the signal in time. In the case of a Gaussian window, the STFT becomes Gabor transform. The STFT spectrogram can be viewed as representing the signal in a dictionary containing truncated sines of different frequencies and time positions, but constant time widths. In contrast to the STFT, which uses a single analysis window, the wavelet transform offers a tradeoff between time and frequency resolution, i.e., it uses short windows at high frequencies and long windows at low frequencies. As a result, the time resolution improves while the frequency resolution degrades - as the analysis frequency increases. When viewing results of the STFT and wavelet transforms, energy density on time-frequency plane is usually used. A more direct approach to obtain an estimation of the time-frequency energy density is the Wigner-Ville distribution (WVD). However, WVD suffers from severe interferences, called cross-terms. Crossterms are the area of a time-frequency energy density estimate that may be interpreted as indicating false presence of signal activity in time-frequency coordinates. Moreover, the WVDs are twodimensional maps; post-processing, such as visual interpretation, to identify certain structures in the signal map is usually needed. This is not desirable for real-time SHM applications.

\subsection{Matching Pursuit Algorithm}

Matching pursuit (MP) algorithm, introduced independently by Mallat and Zhang (1993) and Qian et al. (1992), is a highly adaptive time-frequency signal decomposition and approximation method. The idea of this algorithm it to decompose a function on a set of elementary functions or atoms, selected appropriately from an over-complete dictionary. The MP decomposition procedure can be described as follows (Durka 2007):

1. Find in the dictionary $D$ the first function $g_{\gamma 0}$ that best fits the signal $x$.

2. Subtract its contribution from the signal to obtain the residual $R^{1} x$.

3. Repeat these steps on the remaining residuals, until the representation of the signal in terms of chosen functions is satisfactory.

In the first MP step, the waveform $g_{\gamma 0}$ which best fits the signal $x$ is chosen from dictionary $D$. The fitness is evaluated by inner product. In each of the consecutive steps, the waveform $g_{\gamma n}$ is fitted to the signal $R^{n} x$, which is the residual left after subtracting results of previous iterations, i.e.

$$
\left\{\begin{array}{l}
R^{0} x=x \\
R^{n+1} x=R^{n} x-\left\langle R^{n} x, g_{\gamma n}\right\rangle g_{\gamma n} \\
g_{\gamma n}=\arg \max _{g_{\gamma i} \in D} \mid\left\langle R^{n} x, g_{\gamma i}\right\rangle
\end{array}\right.
$$

In practice we use finite expansions, for example of $N$ iterations, signal $x$ is given by

$$
x=\sum_{n=0}^{N-1}\left\langle R^{n} x, g_{\gamma n}\right\rangle g_{\gamma n}+R^{N} x
$$

Ignore the $N^{\text {th }}$ residual term $R^{N} x$, we have approximated $x$ as

$$
x \approx \sum_{n=0}^{N-1}\left\langle R^{n} x, g_{\gamma n}\right\rangle g_{\gamma n}
$$

The actual outputs of matching pursuit are given in terms of numbers - parameters of the functions or atoms, fitted to the signal:

1. Parameters of basis functions;

2. Plots of the corresponding basis functions in time domain;

3. Two-dimensional blobs representing concentrations of energy density in the time-frequency 
plane, corresponding to functions from the MP expansion, free of cross-terms.

These parameters provide an exact and complete description of the signal structures. Therefore, the analyzed signal can be readily approximated or reconstructed. This provides good synthesis for applications, such as denoising. Also, these numbers can be used directly to identify those functions that correspond to the signal's structures of interest.

\section{GABOR MATCHING PURSUIT}

In principle, the basis functions used for the decomposition can be very general. However, efficient and informative decomposition can be achieved only on a dictionary containing functions reflecting the structure of the analyzed signal. Since Gaussian-type signal achieves the lower bound of the uncertainty inequality, it is natural to choose Gabor functions (Gaussian envelopes modulated by sine oscillations) to construct dictionary, i.e.

$$
g_{\gamma}(t)=K(\gamma) g\left(\frac{t-u}{s}\right) \cos [\omega(t-u)+\phi]
$$

where, $g(t)=e^{-\pi t^{2}}, K(\gamma)$ is chosen such that $\left\|g_{\gamma}\right\|=1$. Hence contribution of each Gabor function to the signal under analysis can be directly calculated. By scaling, translating, and modulating, i.e., varying the $s, u$ and $\omega$ parameters in Eq.(4), Gabor Gaussian functions can describe variety of shapes. For example, pure sine waves and impulse functions can be treated as sinusoidal with very wide and narrow Gaussian modulating windows.

\subsection{State of the Art of Gabor Matching Pursuit}

Mallat and Zhang (1993) applied the MP algorithm using a Gabor dictionary with discretized parameters, i.e.

$$
\gamma(s, u, \omega)=\left(2^{j}, p 2^{j} \Delta u, k 2^{-j} \Delta \omega\right)
$$

where $\Delta u=1 / 2, \Delta \omega=\pi, 0<j<\log _{2}(M), 0 \leq p<M \cdot 2^{-j+1}, 0 \leq k<2^{j+1}, \omega$ is the normalized angular frequency ranging from 0 to $2 \pi$ (sampling frequency), and $M$ denotes the signal length.

To reduce computation effort in each iteration of Eq.(1), Mallat and Zhang (1993) proposed an updating formula derived from Eq.(1) after the vector $g_{\gamma n}$ is selected, i.e.

$$
\left\langle R^{n+1} x, g_{\gamma}\right\rangle=\left\langle R^{n} x, g_{\gamma}\right\rangle-\left\langle R^{n} x, g_{\gamma n}\right\rangle\left\langle g_{\gamma n}, g_{\gamma}\right\rangle
$$

Since $\left\langle R^{n} x, g_{\gamma}\right\rangle$ and $\left\langle R^{n} x, g_{\gamma n}\right\rangle$ are previously stored, only $\left\langle g_{\gamma n}, g_{\gamma}\right\rangle$ needs to be calculated. This particular implementation gives only $M \cdot \log _{2}(M)$ numerical complexity for each iteration.

A variety of discretizing of dictionary parameters, allows for different implementation of MP algorithm. Durka et al (2001) and Durka (2007) randomized the Gabor parameters and formed stochastic dictionary to decompose large amounts of electroencephalogram (EEG) data to eliminate the statistical bias after a careful selection of a subset $D_{a}$ of the potentially infinite dictionary $D_{\infty}$. The choice of $g_{\gamma n}$ in each iteration is performed in two steps:

1. First perform a complete search of the subset $D_{a}$ to find th eparameters $\tilde{\gamma}$ of a function $g_{\tilde{\gamma}}$, giving the largest product with the residuum

$$
\tilde{\gamma}=\arg \max _{g_{\gamma} \in D}\left|\left\langle R^{n} x, g_{\gamma}\right\rangle\right|
$$


2. Secondly, search the neighborhood of the parameter $\tilde{\gamma}$ for a function $g_{\gamma n}$, giving possibly an even larger product $\left|\left\langle R^{n} x, g_{\gamma}\right\rangle\right|$ than $\left|\left\langle R^{n} x, g_{\tilde{\gamma}}\right\rangle\right|$.

Lu and Michaels (2008) used a similar strategy as described in Durka (2007) to decompose ultrasonic signals using Gabor dictionary and applied constrained MP algorithm to identify the change in the PZT transducer data caused by temperature variation for structural health monitoring application. Ferrando and Kolasa (2002) presented two implementations of MP decomposition using Gabor dictionary. In the first implementation, the fixed interval constrains described by Eq.(5) are alleviated. The method allows for greater flexibility in the choice of parameters defining the Gabor dictionary. The second implementation takes advantage of fast Fourier transform algorithm and utilizes a analytical method to optimize the phase parameter $\Phi$, while phase parameter $\Phi$ in Mallat and Zhang (1993) is sub-optimal.. However, it is still within the interval framework of Eq.(5).

\subsection{Simulation Results}

\subsubsection{Excitation Signals}

The excitation signal (Figure 1a) was simulated by the Gabor function in Eq.(4) with

$$
\gamma(s, u, \omega, \phi)=(1 / f, 3 / f, 2 \pi f, 0)
$$

where frequency $f=350 \mathrm{kHz}$ at which the $\mathrm{S}_{0}$ Lamb wave is dominant, moderately dispersive, with a group velocity $c_{g r}=4988 \mathrm{~m} / \mathrm{s}$ on $3-\mathrm{mm}$ aluminum plate (Figure $1 \mathrm{~b}$ ).

\subsection{2. $\quad$ Reception Signals}

Assuming the transmission wave is reflected by three ideal reflectors, the reflected wave (Figure 2a) can be simulated as

$$
f(t)=\operatorname{IFFT}\left\{G T B(\omega)\left[e^{-i k_{s}(\omega) x_{1}}+e^{-i k_{s}(\omega) 2 x_{2}}+e^{-i k_{s}(\omega) 2 x_{3}}\right]\right\}
$$

where IFFT $\{\cdot\}$ denotes the inverse fast Fourier transform; GTB $(\omega)$ denotes Fourier transform of the excitation signal based on Gabor function; $x_{1}=100 \mathrm{~mm}, x_{2}=200 \mathrm{~mm}, x_{3}=350 \mathrm{~mm}$ are locations of the three reflectors; and $k_{\mathrm{s}}(\omega)$ are $\mathrm{S}_{0}$ mode wavenumbers obtained by solving Rayleigh-Lamb wave equation. A reflected wave contaminated by uniform noise at signal to noise ratio $S N R=1.6 \mathrm{~dB}$ is also simulated, as shown in Figure 2b. In both plots, due to the dispersion effect, the wave packets become more and more spreading out in time domain as the propagation distance increases.

The reception signals are processed by $L_{2}$ normalization before decomposition in the format of

$$
\bar{x}=\frac{x}{\sqrt{\sum x_{i}^{2}}}
$$

where $\bar{x}$ denotes the normalized signal and $x$ is the signal before normalization. 
(a)

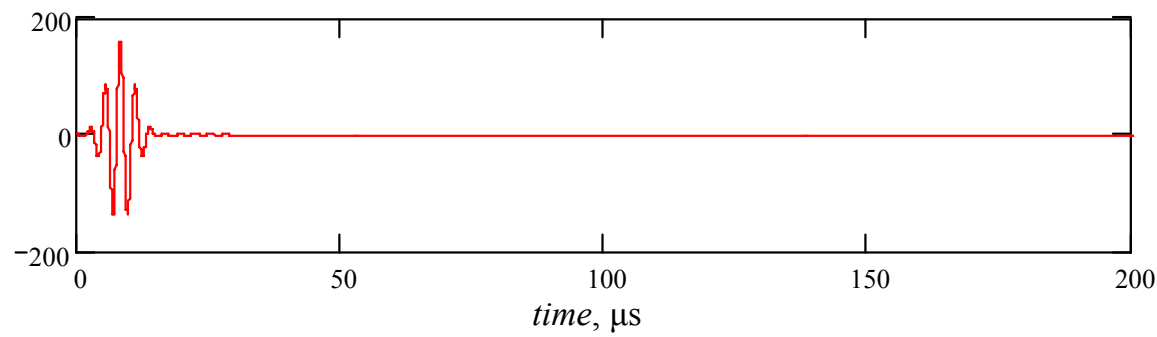

(b)

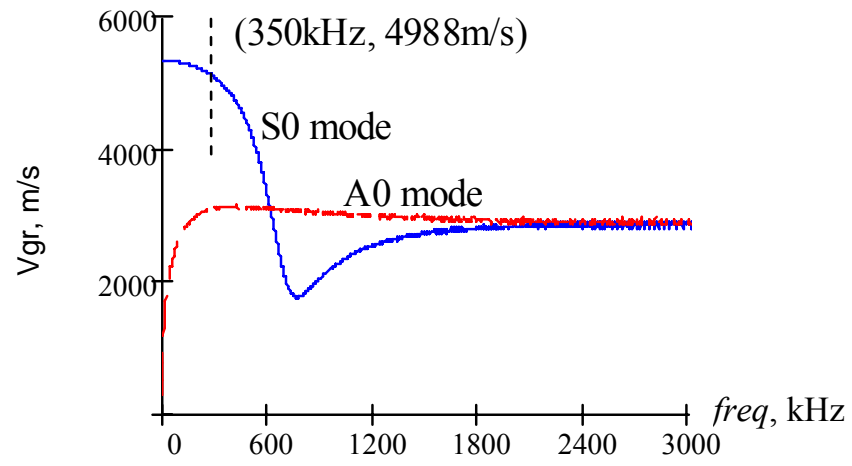

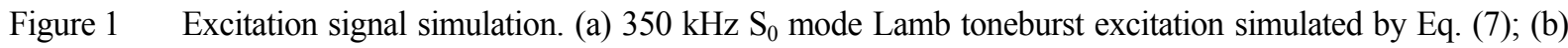
group velocity plot of Lamb wave modes $\mathrm{A} 0$ and $\mathrm{S} 0$ on a 3-mm aluminum plate.

(a)
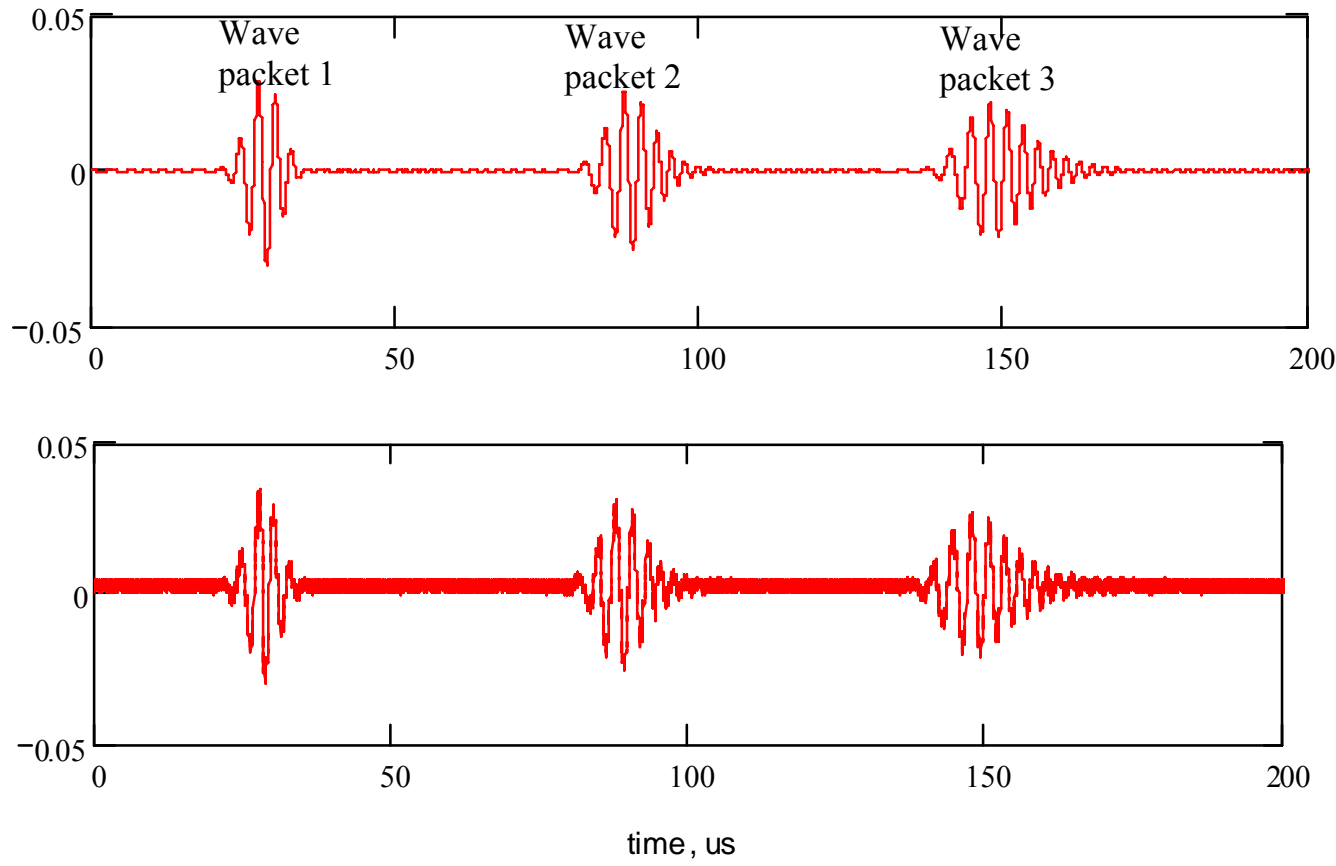

Figure 2 Simulated reception signals. (a) Clean signal; (b) Noisy signal with $\mathrm{SNR}=1.6 \mathrm{~dB}$

\subsubsection{Gabor dictionary matching pursuit decomposition}

In our work, MP algorithm presented in Ferrando and Kolasa (2002) was implemented in Visual studio 2005 and tested to decompose/approximate Lamb waves and extract the wave parameters. Stop criteria of MP decomposition is set to either (1) maximum iteration number at $300\left(N_{\max }=300\right)$ or (2) 
residual energy $d<0.01$, whichever is reached first. The residual energy $d$ is defined as

$$
d=\sqrt{\sum_{i}\left(x-\sum_{n=0}^{N-1}\left\langle R^{n} x, g_{\gamma n}\right\rangle\right)^{2}}
$$

In the clean signal example, the MP decomposition stopped after 45 iterations when the residual energy satisfied the stop criteria (2). The residual energy at each iteration is plotted in Figure 3 . The procedure took about four minutes for the sampled signal of $M=2^{14}$ data points.

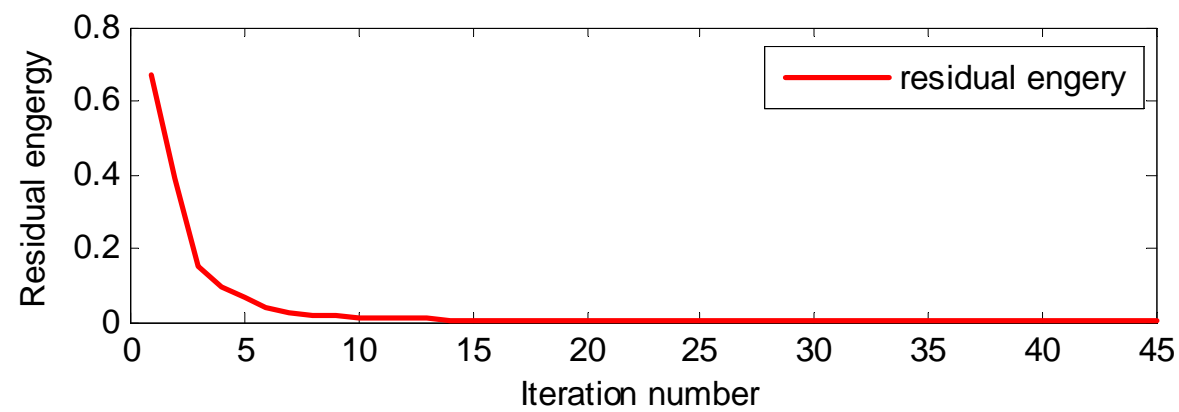

Figure 3 Residual energy at each iteration during the Gabor matching pursuit of the clean signal

Figure 4 is the reconstructed signal using all the 45 decomposed dictionary atoms. Comparison between Figure 2a and Figure 4 reveals that the original wave can be fully reconstructed using the decomposed atoms.

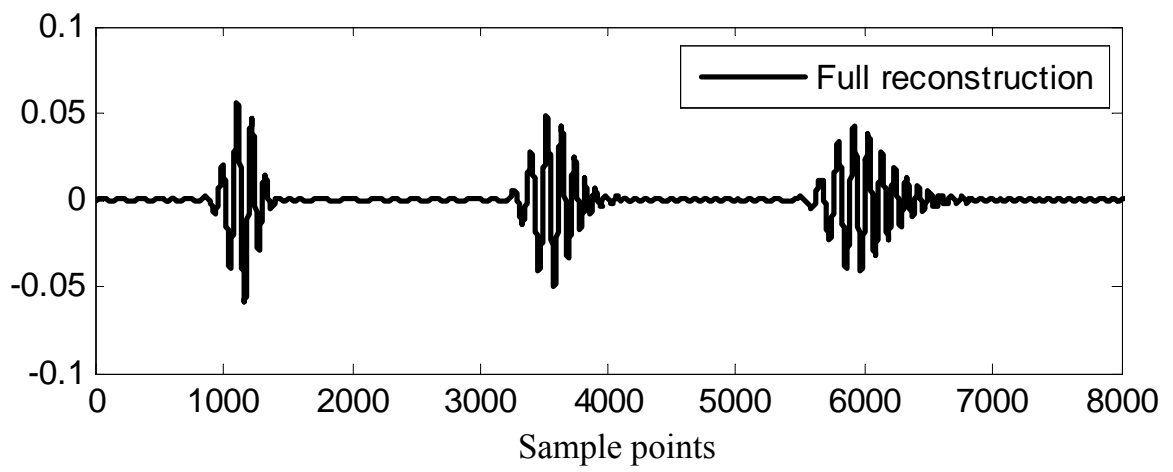

Figure 4 Signal approximated with all the 45 decomposed Gabor dictionary atoms

Figure 5a shows the reconstructed wave using only the first three decomposed atoms (\#0, \#1, \#2). As given in Figure 5b where the first 10 decomposed atoms from dictionary are plotted, the first three dictionary atoms possess most of the energy of the signal.

An estimation of the first three wave packets parameters is tabulated in Table 1 and compared to their actual values. For the first decomposed vector which actually is a non-dispersive wave packet, the estimated parameters by Gabor matching pursuit approach are very close to their actual values. As the wave packets become dispersive, for example the second and the third atom in the dictionary, an increased deviation of the estimated parameters from their actual values was observed.

\subsubsection{Effects of noise in the signal}

To check the additive noise effects on the algorithm, the contaminated signal with uniform noise shown in Figure 2b was analyzed with Gabor MP algorithm. After normalization, the noisy signal was processed as described above. The residual energy plot is given in Figure 6. The decomposition process stopped with criteria (1) was satisfied when iteration number reached 300. Compared with the residual energy plot of the clean signal in Figure 3, we see the presence of noise increases the decomposition efforts that the residual energy $\mathrm{d} \approx 0.07$ in the end, much larger than the clean signal 
case. However, the presence of noise does not affect the accuracy of the extracted wave parameters, such as time-of-flight, central frequency, etc. The parameters estimation of the first three wave packets by the decomposition in this case is identical to the case of a clean signal.

The first ten decomposed atoms of the noisy signal are presented in Figure 7b. Due to the presence of noise, the first decomposed vector does not resemble any of the wave packets in the raw signal. However, we have found out that this can be avoided by careful selection of the time scale of basis functions. Decomposed atoms \#1, \#2 and \#3 were used to reconstruct the signal, which is given in Figure 7a.

(a)

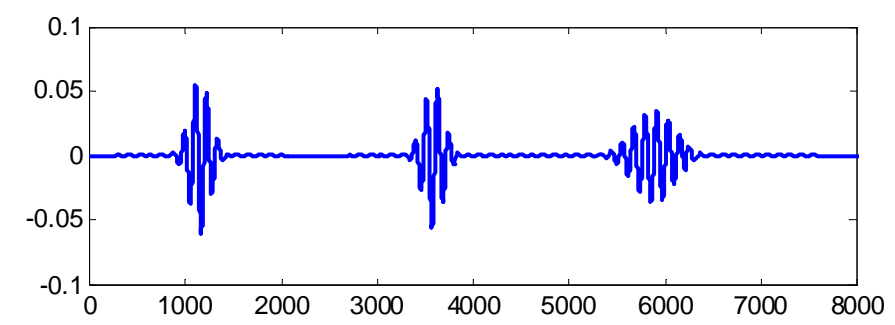

(b)

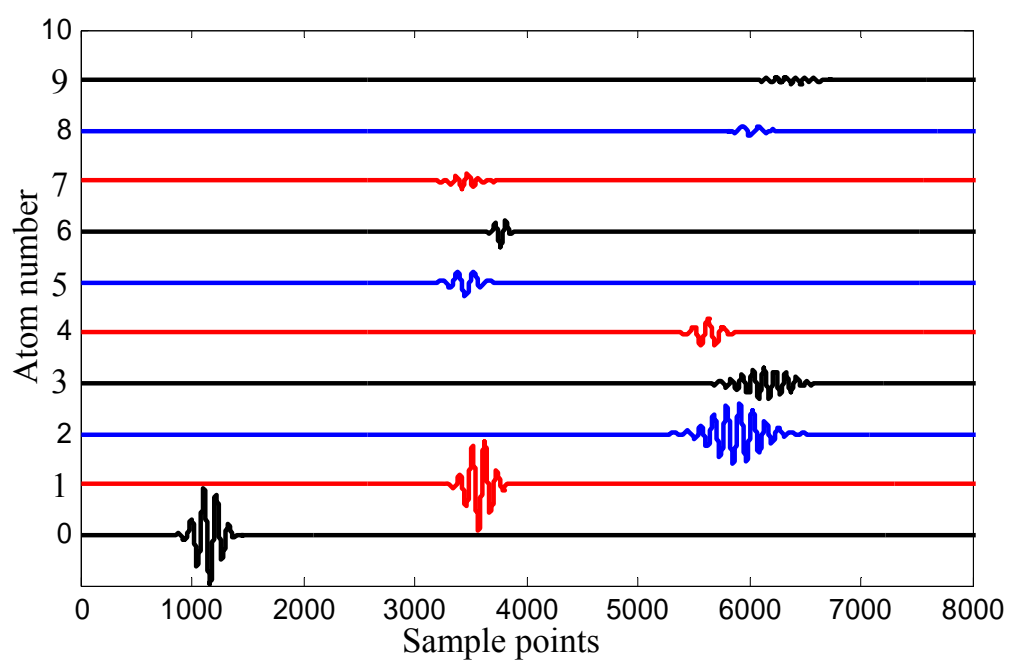

Figure 5 Signal approximated with the first 3 decomposed Gabor dictionary atoms. (a) The partially reconstructed signal; (b) the first 10 decomposed dictionary atoms

Table 1 Wave packet parameter estimation by Gabor matching pursuit in comparison with actual values

\begin{tabular}{ccccccc}
\hline & \multicolumn{2}{c}{ wave packet 0 } & \multicolumn{2}{c}{ wave packet 1 } & \multicolumn{2}{c}{ wave packet 2 } \\
\hline & MP & actual & MP & actual & MP & actual \\
\hline TOF $(\mu \mathrm{s})$ & 20.23 & 20.05 & 81.03 & 80.19 & 138.63 & 140.34 \\
$\mathrm{f}(\mathrm{kHz})$ & 351.56 & 351.56 & 351.58 & 351.560 & 341.38 & 351.56 \\
\hline
\end{tabular}

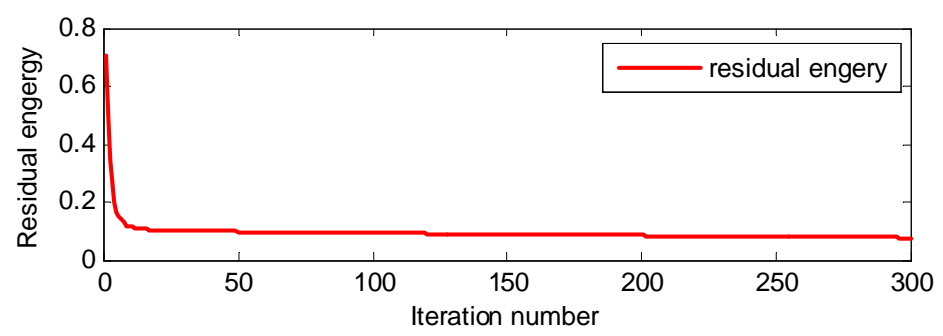

Figure 6 Residual energy plot of uniform noise signal processed by Gabor matching pursuit 
(a)

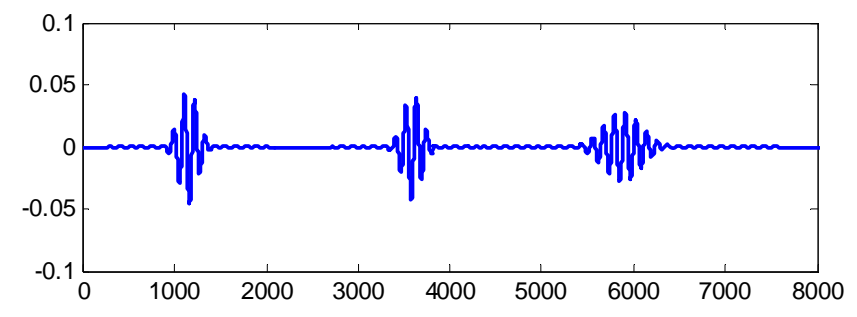

(b)

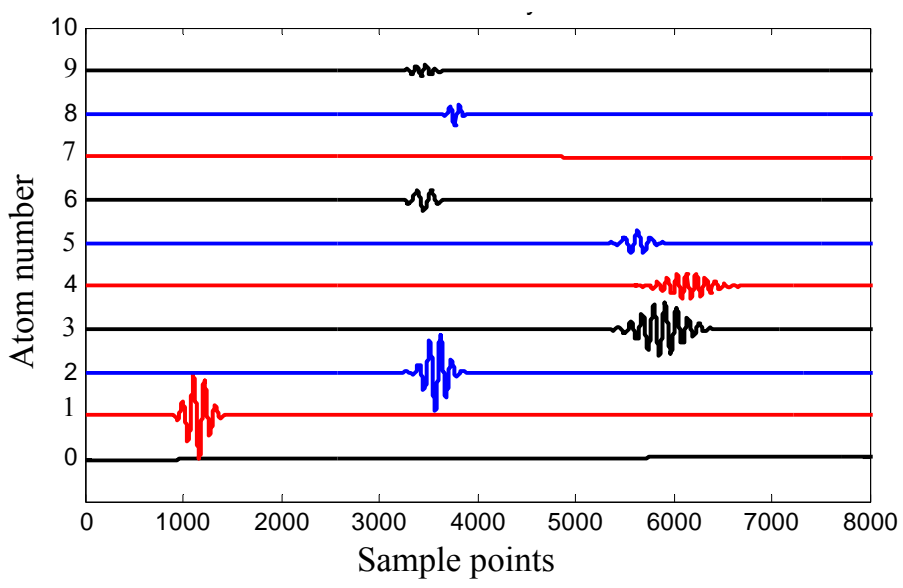

Figure 7 Signal approximated with the \#1 to \#3 decomposed Gabor dictionary atoms of a noisy signal. (a) The partially reconstructed signal; (b) the first 10 decomposed dictionary atoms

\section{CHIRPLET MATCHING PURSUIT}

As it can be seen both from Figure $5 \mathrm{~b}$ and Figure $7 \mathrm{~b}$, the decomposed atoms from Gabor dictionary are typically an even-symmetric toneburst because the associated Gabor dictionary basis functions exhibit symmetric time-domain behavior. This is problematic for decomposing signal with asymmetric features, such as dispersed or asymmetric signals which occur frequently in guided wave propagation. To decompose such signals, matching pursuit using other dictionaries, such as damped sinusoidal and chirplet, may be needed. In our application, we use the matching pursuit algorithm based on chirplet dictionary to take the dispersion effect into account.

Gribonval (2001) introduced a fast MP algorithm using Gaussian chirplet implemented as a toolbox in the LASTWAVE (2008). The algorithm is based on post-processing of the Gabor atom and aimed at optimizing the chirp rate. Hong et al. (2006) used matching pursuit based on chirplet dictionary with quadratic group delay to decompose a longitudinal wave in a rod. Raghavan and Cesnik (2007) used LASTWAVE to analyze Lamb waves and correlated chirp-rates to different Lamb wave modes. The correlation procedure was performed in two steps. In the first step, matching pursuit was performed on each simulated mode of Lamb wave at possible time and frequency centers to extract the chirp rates and generate a database of chirp-rates for different mode Lamb waves. In the second step, matching pursuit was performed on the wave under analysis to extract chirp rate and then correlated the chirp rate to the database to identify wave mode. However, since the knowledge of time center needed in the first step is usually unknown, the procedure is not trivial and a large database has to be generated.

In our research, matching pursuit based on Gaussian chirplet dictionary was explored to decompose Lamb waves using LASTWAVE software package. The decomposition results were analyzed and compared to the results obtained with Gabor dictionary. In addition, a simple but effective way to identify low-frequency Lamb wave modes using the extracted chirp rates has been 
developed.

\subsection{Gaussian Chirplet}

Gaussian chirplet is a Gaussian smoothed linear chirp (with quadratic phase) that can be expressed as

$$
g_{\gamma\left(s, u, \omega_{0}, c\right)}(t)=\frac{1}{\sqrt{s}} g\left(\frac{t-u}{s}\right) \mathrm{e}^{i\left(\omega_{0}(t-u)+\frac{c}{2}(t-u)^{2}\right)}
$$

Defining phase $\phi(t)=\omega_{0}(t-u)+\frac{c}{2}(t-u)^{2}$, we have instant frequency of the Gaussian chirplet as

$$
\omega_{i n s t}=\frac{d \phi(t)}{d t}=\omega_{0}+c(t-u)
$$

When chirp rate $c=0$, Gaussian chirplet is degraded to a Gabor function which has a constant instant frequency. When chirp rate $c>0$, the instant frequency increases linearly with time, indicating a positively chirped pulse. When chirp rate $c<0$, the instant frequency decreases linearly with time, indicating a negatively chirped pulse accordingly. Defining $\alpha=2 \pi / s^{2}, \beta=c$, Eq.(11) can be written as

$$
g_{\gamma(s, u, \omega, c)}(t)=\sqrt[\frac{1}{4}]{\frac{2 \pi}{\alpha}} \mathrm{e}^{\left[-\frac{(\alpha-i \beta)}{2}(t-u)^{2}\right]} \mathrm{e}^{i \omega_{0}(t-u)}
$$

Fourier transform of Eq.(13) using time delay, frequency translation and Gaussian function Fourier transform pairs, yields

$$
G(\omega)=\sqrt[\frac{1}{4}]{\frac{2 \pi}{\alpha}} \sqrt{\frac{2 \pi}{\alpha-i \beta}} \mathrm{e}^{-\frac{\left(\omega-\omega_{0}\right)^{2}}{2(\alpha-i \beta)}} \mathrm{e}^{-i\left(\omega-\omega_{0}\right) u}
$$

Separating the imaginary and real parts of Eq.(14), we are able to define the phase and group delay as

$$
\begin{aligned}
& \quad \phi(\omega)=\frac{\beta\left(\omega-\omega_{0}\right)^{2}}{2\left(\alpha^{2}+\beta^{2}\right)}+u\left(\omega-\omega_{0}\right)-\tan ^{-1}\left(\frac{\beta}{2 \alpha}\right) \\
& \text { Phase delay } \\
& \text { Group delay }
\end{aligned}
$$

The group delay in Eq.(16) shows that the group delay of a linear chirp varies linearly with the frequency $\omega$. The group delay derivative w.r.t. $\omega$ can be expressed as

$$
\frac{d \tau G(\omega)}{d \omega}=\frac{\beta}{\alpha^{2}+\beta^{2}}=\frac{c}{\alpha^{2}+c^{2}}
$$

The sign of the derivative (group delay slope) is determined by the sign of chirp rate, $c$, as shown in (17)Eq.

\subsection{Lamb Wave Mode Identification Using Chirplet Decomposition}

For a propagating wave at distance $x=x_{0}$, its expression in frequency domain can be approximated as

$$
\left.S(x, \omega)\right|_{x=x_{0}}=\left.S(x, \omega)\right|_{x=0} e^{-i k(\omega) x_{0}}
$$

Then its group delay can be represented as 


$$
\begin{aligned}
\tau G(\omega) & =\frac{d \phi(\omega)}{d \omega}=\frac{d\left[k(\omega) x_{0}\right]}{d \omega} \\
& =\frac{x_{0}}{d \omega / d k(\omega)}=\frac{x_{0}}{c_{g r}(\omega)}
\end{aligned}
$$

Group delay versus frequency curves can be obtained from Eq.(19), as plotted in Figure 8 for $\mathrm{S}_{0}$ and $\mathrm{A}_{0}$ Lamb wave modes on a $3 \mathrm{~mm}$ aluminum plate. We can see that the group delays of $\mathrm{S}_{0}$ and $\mathrm{A}_{0}$ modes are not in linear relationship with frequency. This implies that if we use the linear chirp dictionary to decompose a single Lamb wave packet, though the wave packet will still be decomposed into a number of dictionary atoms, the residual energy might diminish faster as compared to the case of using Gabor dictionary, which has a constant group delay. In other words, fewer atoms might be needed to decompose a Lamb wave packet when using a Gaussian chirp dictionary than when using a Gabor dictionary.

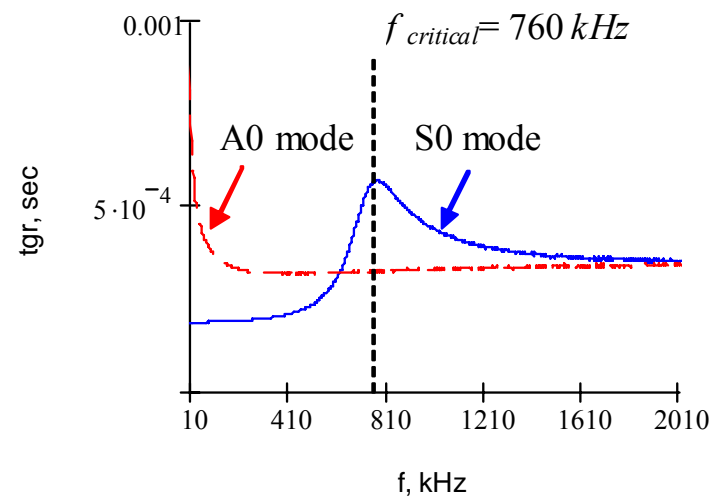

Figure 8 Group velocity of S0 and A0 Lamb wave modes on a 3mm aluminum plate

As aforementioned, the decomposed Gaussian chirplet atoms have an additional parameter, the chirp rate $c$, which can be correlated with the mode of Lamb wave. For a low frequency SHM applications below several hundred $\mathrm{kHz}$ such as the example presented in Figure 8, below the critical frequency $f_{\text {critical }}=760 \mathrm{kHz}$, only two modes exist, $\mathrm{A}_{0}$ and $\mathrm{S}_{0}$; and the group delay slope of the $\mathrm{A}_{0}$ mode is always negative; while the group delay slope of the $\mathrm{S}_{0}$ mode is always positive. Therefore, based on the Eq. (17), one can correlate the sign of chirp rate $c$ with the two widely used Lamb wave modes that usually are excited below certain critical frequency point, which we call "mode identification". If the decomposed chirp atom satisfies the condition $c>0$, the wave packet being decomposed is $\mathrm{S}_{0}$ mode; if the decomposed chirp atom has the case of $c<0$, the wave packet is $\mathrm{A}_{0}$ mode.

\subsection{Chirplet decomposition of SO Lamb wave}

MP decomposition using chirplet dictionary was applied to a simulated $\mathrm{S}_{0}$ mode Lamb wave shown previously in Figure 2a. Wigner-Ville distribution of the simulated signal is plotted in Figure 9. As we predicted and expected, S0 mode wave packets should have positive chirp rates. In addition, due to dispersion, as the wave propagates along the structure, the wave spreads out in the time domain. The spreading will cause a decrease in the chirp rates according to the definition of chirp rate (Eq.(12)). The positive and decreasing chirp rates of each wave packets in this S0 mode signal can be seen clearly in the WVD plot as indicated, confirming our theoretical prediction and verified that we can determine the mode of the wave being decomposed by evaluating the sign of the chirplet rate. 


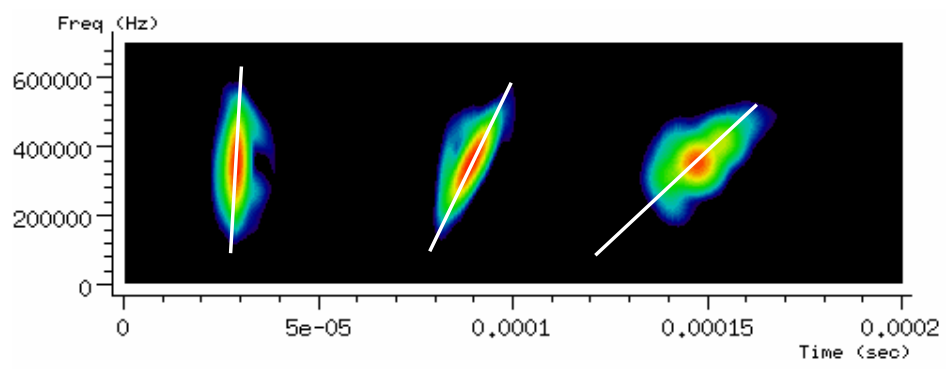

Figure 9 Wigner-Ville distribution of simulated S0 Lamb wave.

The estimation of the first three wave packets parameters is tabulated in Table 2. Comparing the data in Table 1 using Gabor MP and Table 2 using Chirplet MP, we found the results are very close to each other. However, chirplet approach shows slightly better decomposition result in that fewer atoms are needed to reconstruct the simulated $\mathrm{S}_{0}$ mode wave when using chirplet dictionary, as revealed by the plots of residual energy vs. iteration number in Figure 10.

Table 2 Wave packet parameter estimation by Chirplet matching pursuit in comparison with actual values

\begin{tabular}{ccccccc}
\hline & \multicolumn{2}{c}{ wave packet 1 } & \multicolumn{2}{c}{ wave packet 2 } & \multicolumn{2}{c}{ wave packet 3 } \\
\hline & MP & actual & MP & actual & MP & actual \\
\hline $\mathrm{TOF}(\mu \mathrm{s})$ & 20.23 & 20.05 & 81.03 & 80.19 & 138.63 & 140.34 \\
$\mathrm{f}(\mathrm{kHz})$ & 349.92 & 351.56 & 353.24 & 351.56 & 351.56 & 351.56 \\
\hline
\end{tabular}

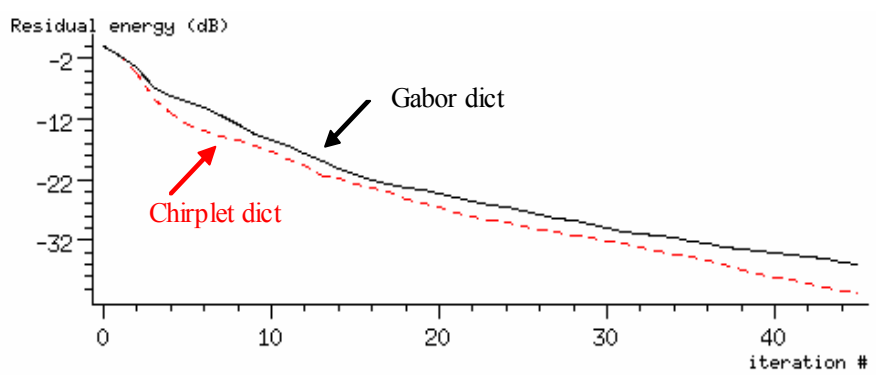

Figure 10 Residual energy plots for both chirplet and Gabor dictionary decomposition w.r.t. iteration number

\section{CONCLUSIONS}

Matching pursuit (MP) is an adaptive signal decomposition technique and can be easily implemented and automated to process Lamb waves, such as denoising, wave parameter estimation, and feature extraction for SHM applications. This paper explored matching pursuit algorithm based on Gabor and chirplet dictionaries to decompose/approximate Lamb waves and extract wave parameters. While Gabor dictionary based MP is optimal for decomposing symmetric signals, chirplet dictionary based MP is able to decompose asymmetric signals, e.g., dispersed Lamb wave. The extracted parameter from the chirplet MP, chirp rate $c$, can be used to correlate with and identify low-frequency Lamb wave mode. We have found out that a wave with positive sign of chirp rate $(c>0)$ denotes $\mathrm{S}_{0}$ mode Lamb wave and one with negative sign of chirp rate $(c<0)$ denotes $\mathrm{A}_{0}$ mode Lamb wave. A simulation has been conducted to verify the $\mathrm{S}_{0}$ mode identification using chirplet dictionary MP and compared with Gabor approach in terms of residual energy and iteration numbers.

The next stage we will verify mode identification the $\mathrm{A}_{0}$ mode and mixed modes situations with the chirplet decomposition. Further work will also be done on Gaussian-windowed nonlinear chirp (with cubic phase) atoms which has the potential to fully describe the nonlinear group delay feature of Lamb waves. 


\section{ACKNOWLEDGMENTS}

The financial support of National Science Foundation under Grant \#CMS-0408578 is thankfully acknowledged.

\section{REFERENCES}

Durka, P.J.; Ircha, D.; Blinowska, K.J. (2001) "Stochastic time-frequency dictionaries for matching pursuit." IEEE Trans. Signal Processing, vol. 49, pp. 507-510

Durka, P.J. (2007), Matching Pursuit and Unification in EEG analysis, Artech House

Edalati, K.; Kermani, A.; Seiedi, M.; Movafeghi,M. (2005) "Defect Detection in Thin Plates by Ultrasonic Lamb Wave Techniques", the 8th International Conference of the Slovenian Society for Non-Destructive Testing, Application of Contemporary Non-Destructive Testing in Engineering, September 1-3, 2005, Portorož, Slovenia, pp. 35-43

Ferrando, S.E.; Kolasa, L.A. (2002) “Algorithm 820: A Flexible Implementation of Matching Pursuit for Gabor Functions on the Interval", ACM Trans. On Mathematical Software, Vol. 28, No. 3, pp. 337 - 353

Giurgiutiu, V. (2008) Structural Health Monitoring with Piezoelectric Wafer Active Sensors, Academic Press, ISBN 9780120887606

Gribonval, R. (2001) "Fast matching pursuit with a multiscale dictionary of Gaussian chirps", IEEE Trans. Signal Process, vol. 49, pp. 994-1001

Hong, J.-C.; Sun, K.H.; Kim, Y.Y. (2006) "Waveguide Damage Detection by the Matching Pursuit Approach Employing the Dispersion-based Chirp Functions," IEEE Trans. On Ultrasonics, Ferroelectrics, and Frequency Control, Vol. 53, No. 3, pp. 592-605

Lankhorst, M.M. (1996) Genetic algorithms in data analysis, dissertation, ISBN 90-367-0559-2, pp. 73-93 (http://dissertations.ub.rug.nl/faculties/science/1996/m.m.lankhorst/)

LASTWAVE (2008), www.cmap.polytechnique.fr/ bacry/LastWave

Lu, Y.; Michaels, J.E. (2008) "Numerical Implementation of Matching Pursuit for the analysis of Complex Ultrasonic Signals", IEEE Trans. On Ultrasonics, Ferroelectrics, and Frequency Control, Vol. 55, No. 1, pp. $173-182$

Mallat, S.; Zhang, Z. (1993) "Matching pursuits with time-frequency dictionaries," IEEE Trans. Signal Processing, vol. 41, pp. 3397-3415

Qian, S.; Chen, D.; Chen, K. (1992) "Signal approximation via data-adaptive normalized Gaussian function and its applications for speech processing," Proc. ICASSP'92, San Francisco, CA, Mar. 23-26, 1992, pp. 141144.

Raghavan, A.; Cesnik, C. (2007) "Guided-wave signal processing using chirplet matching pursuits and mode correlation for structural health monitoring”, Smart Materials and Structures, vol. 16, pp. 355-366

Thompson, D.O. and Chimenti, D.E., Eds. (2002) Review of Progress in Quantitativ4 Nondestructive Evaluation: AIP Conf. Proc., July 12-19, 2002, Bellingham, WA, Vol. 615, Chaps, 2C and 7.

Pan, E.; Rogers, J.; Datta, S.K.; Shah, A.H., (1999) "Mode selection of guided waves for ultrasonic inspection of gas pipelines with thick coating", Mechanics of Materials, 31, pp. 165-174.

Rose, J.L,, Rajana, K.M., and Hansch, M.K.T., Ultrasonic guided 